\title{
Matrix structure of unified mathematical model of electric AC machines at control
}

\author{
Rauf Mustafayev*, Nurali Yusifbayli, Laman Hasanova \\ Azerbaijan Scientific-Research and Designed-Prospecting Institute of Energetics, Baku, Azerbaijan
}

\begin{abstract}
The matrix structure of the equations of a generalized electric alternating current machine is proposed, which, based on the Parke equations, is written in the coordinate axes of the machines rotating with the rotor speed. In the matrix structure, the column matrices of the derivatives of the stator, excitation and rotor windings are equal to the product of diagonal matrices consisting of the machine parameters and the column matrices of the flux links themselves and the sum of the matrix columns of the control parameters which are the matrix columns of the stator voltage, excitation voltage, and rotor voltage. It is shown that the matrix structure of a generalized controlled AC machine is transformed into mathematical models of almost all encountered AC electric machines, namely, into a synchronous machine with two excitation windings - a longitudinal and a transverse one; in a synchronous machine with a longitudinal field winding (classic); in an asynchronous machine with a squirrel-cage rotor; into an asynchronous machine with a phase rotor. It has been shown that the matrix structure includes the controls of these machines both from the stator and from the rotor. On the stator side for synchronous machines, it is a frequency control that regulates both the amplitude and frequency of the applied voltage, and on the rotor side, a constant voltage control is supplied to the longitudinal and transverse windings. For asynchronous machines, the stator and rotor are frequency-controlled. The following are examples of frequency control of an asynchronous machine both from the stator and from the rotor.
\end{abstract}

\section{Introduction}

When designing complex electromechanical devices for such, for example, systems as "wind-power engineering", "small hydropower engineering", etc., which can contain controlled electric machines of different types, it is required not only optimally join electric machine with mechanical one, but in a number of cases to optimize the choice and the type itself of the controlled electric machine.

The equations of a generalized electric machine are well known, which are given, for example, in [1]. It should be noted that these equations more reveal the principle of construction of electromagnetic and electrical connections in electric machine, for practical use they must be transformed taking into account the type of electric machine and the form of writing of their equations.

In addition, the controllability principles and transient processes in individual AC electric machines are studies in papers of famous scientists $[2 ; 3 ; 4 ; 5 ; 6 ; 7 ; 8]$.

\section{Materials and methods}

The above circumstances predetermine the creation of a universal mathematical model of a controlled alternating current (AC) electric machine, which, remaining structurally unchanged, would allow for studying all modes of operation of AC electric machines used in practice: synchronous machines with electromagnetic excitation and permanent magnets, including frequencycontrolled ones; asynchronous machines with shortcircuited and phase-wound rotor, including frequencycontrolled ones.

Here, Park's equations, written in axes rotating at the rotor speed of $\omega_{r}$, were taken as the basis of mathematical model of electric AC machines, and the machine has two stator windings and four rotor windings. In contrast with these equations, not angle $\theta$ between the rotor axis, rotating at the rotor speed of $\omega_{r}$, and the synchronous axis, rotating at the synchronous speed of $\omega_{s}$, but the

\footnotetext{
* Corresponding author: mustafayevri@mail.ru
} 
angle between the rotor axis and fixed axis, hereinafter signed as angle $\alpha$, is selected as a power angle. Thus, the rotor speed is equal to $\omega_{r}$, where $p$ - differentiation symbol, $\tau-$ synchronous time equal to $\tau=\omega_{s} \cdot t=314 \cdot \mathrm{t}$. In addition, the equations of machines are written in flux linkages.

In this case the equations of controlled electric $\mathrm{AC}$ machines can be written in cell wise-matrix form, which is represented in the form.

$$
\left[\begin{array}{l}
p \boldsymbol{\Psi}_{s} \\
p \boldsymbol{\Psi}_{f} \\
p \boldsymbol{\Psi}_{r}
\end{array}\right]=\left[\begin{array}{lll}
\boldsymbol{A}_{s 1} & \boldsymbol{A}_{s 2} & \boldsymbol{A}_{s 3} \\
\boldsymbol{B}_{f 1} & \boldsymbol{B}_{f 2} & \boldsymbol{B}_{f 3} \\
\boldsymbol{C}_{r 1} & \boldsymbol{C}_{r 2} & \boldsymbol{C}_{r 3}
\end{array}\right] \cdot\left[\begin{array}{l}
\boldsymbol{\Psi}_{s} \\
\boldsymbol{\Psi}_{f} \\
\boldsymbol{\Psi}_{r}
\end{array}\right]+\left[\begin{array}{l}
\boldsymbol{U}_{s} \\
\boldsymbol{U}_{f} \\
\boldsymbol{U}_{r}
\end{array}\right]
$$

Column matrices are the essence of a vector with projections on $d, q$ axes:

- derivatives of flux linkages of stator, field and rotor windings:

$$
p \boldsymbol{\Psi}_{s}=\left[\begin{array}{c}
p \boldsymbol{\Psi}_{d s} \\
p \boldsymbol{\Psi}_{q s}
\end{array}\right], \quad p \boldsymbol{\Psi}_{f}=\left[\begin{array}{c}
p \boldsymbol{\Psi}_{d f} \\
p \boldsymbol{\Psi}_{q f}
\end{array}\right], p \boldsymbol{\Psi}_{r}=\left[\begin{array}{c}
p \boldsymbol{\Psi}_{d r} \\
p \boldsymbol{\Psi}_{q r}
\end{array}\right]
$$

- flux linkages $\Psi_{s}, \Psi_{r}, \Psi_{f}$ themselves :

$$
\boldsymbol{\Psi}_{s}=\left[\begin{array}{l}
\boldsymbol{\Psi}_{d s} \\
\boldsymbol{\Psi}_{q s}
\end{array}\right], \boldsymbol{\Psi}_{f}=\left[\begin{array}{l}
\boldsymbol{\Psi}_{d f} \\
\boldsymbol{\Psi}_{q f}
\end{array}\right], \boldsymbol{\Psi}_{r}=\left[\begin{array}{l}
\boldsymbol{\Psi}_{d r} \\
\boldsymbol{\Psi}_{q r}
\end{array}\right]
$$

-voltages of stator, field and rotor windings (control actions):

$$
\boldsymbol{U}_{s}=\left[\begin{array}{c}
U_{d s} \\
U_{q s}
\end{array}\right], \boldsymbol{U}_{f}=\left[\begin{array}{c}
U_{d f} \\
U_{q f}
\end{array}\right], \boldsymbol{U}_{r}=\left[\begin{array}{c}
U_{d r} \\
U_{q r}
\end{array}\right]
$$

Matrices $\boldsymbol{A}_{s 1}, \boldsymbol{A}_{s 2}, \boldsymbol{A}_{s 3}, \boldsymbol{B}_{f 1}, \boldsymbol{B}_{f 2}, \boldsymbol{B}_{f 3}$, as well as $\boldsymbol{C}_{r 1}, \boldsymbol{C}_{r 2}$, $\boldsymbol{C}_{r 3}$ are diagonal matrices, which are represented in the form:

$$
\begin{aligned}
& \boldsymbol{A}_{s 1}=\left[\begin{array}{cc}
-r_{s} \cdot k_{d s} & -\omega_{r} \\
\omega_{r} & -r_{s} \cdot k_{q s}
\end{array}\right] ; \boldsymbol{A}_{s 2}=\left[\begin{array}{cc}
-r_{s} \cdot k_{d s f} & 0 \\
0 & -r_{s} \cdot k_{q s f}
\end{array}\right] ; \\
& \boldsymbol{A}_{s 3}=\left[\begin{array}{cc}
-r_{s} \cdot k_{d s r} & 0 \\
0 & -r_{s} \cdot k_{q s r}
\end{array}\right] . \\
& \boldsymbol{B}_{f 1}=\left[\begin{array}{cc}
-r_{d f} \cdot k_{d s f} & 0 \\
0 & -r_{q f} \cdot k_{q s f}
\end{array}\right] ; \boldsymbol{B}_{f 2}=\left[\begin{array}{cc}
-r_{d f} \cdot k_{d f} & 0 \\
0 & -r_{q f} \cdot k_{q f}
\end{array}\right] ; \\
& \boldsymbol{B}_{f 3}=\left[\begin{array}{cc}
-r_{d f} \cdot k_{d f r} & 0 \\
0 & -r_{q f} \cdot k_{q f r}
\end{array}\right] ; \\
& \boldsymbol{C}_{r 1}=\left[\begin{array}{cc}
-r_{d r} \cdot k_{d s r} & 0 \\
0 & -r_{q r} \cdot k_{q s r}
\end{array}\right] ; \boldsymbol{C}_{r 2}=\left[\begin{array}{cc}
-r_{d r} \cdot k_{d f r} & 0 \\
0 & -r_{q r} \cdot k_{q f r}
\end{array}\right] ; \\
& \boldsymbol{C}_{r 3}=\left[\begin{array}{cc}
-r_{d r} \cdot k_{d r} & 0 \\
0 & -r_{q r} \cdot k_{q r}
\end{array}\right] .
\end{aligned}
$$

First matrix $\boldsymbol{A}_{s 1}$ can be represented in the form:

$$
\boldsymbol{A}_{s 1}=\boldsymbol{A}_{s 11}+\boldsymbol{A}_{s 1 \omega}=\left[\begin{array}{cc}
-r_{s} \cdot k_{d s} & 0 \\
0 & -r_{s} \cdot k_{q s}
\end{array}\right]+\left[\begin{array}{cc}
0 & -\omega_{r} \\
\omega_{r} & 0
\end{array}\right]
$$

Since $\omega_{r}$ - rotor speed of AC machine is a scalar value, then $\boldsymbol{A}_{s 1 \omega}$ can be represented in the form:

$$
\boldsymbol{A}_{s 1 \omega}=\left[\begin{array}{cc}
0 & -1 \\
1 & 0
\end{array}\right] \cdot \omega_{r}=-\boldsymbol{J} \cdot \omega_{r}
$$

where $\boldsymbol{J}$-matrix orthogonal to unity matrix $\boldsymbol{E}$, i.e. $\left(\boldsymbol{J}^{2}=-\right.$ $\boldsymbol{E})$, by analogy with complex unit $j^{2}=-1$.

Besides equations (1), it is necessary to take into account equations of motion with motive moment $m_{t}$ and electromagnetic moment $m_{e m}$ :

$$
\left.\begin{array}{l}
T_{j} \cdot p \omega_{r}=m_{t}-m_{e m} \\
m_{e m}=\left(k_{q s}-k_{d s}\right) \cdot \Psi_{d s} \cdot \Psi_{q s}+k_{q s f} \cdot \Psi_{d s} \cdot \Psi_{q f}+ \\
+k_{q s r} \cdot \Psi_{d s} \cdot \Psi_{q r}-k_{d s f} \cdot \Psi_{q s} \cdot \Psi_{d f}-k_{d s r} \cdot \Psi_{q s} \cdot \Psi_{d r}
\end{array}\right\}
$$

Thus, equations (1) and (2) form a universal mathematical model of electric AC machines. In addition to the equation, the definition of active and reactive powers is of interest for $m_{e m}$ :

$$
\left.\begin{array}{c}
p_{e m}=k_{d s} \cdot U_{d s} \cdot \Psi_{d s}+k_{d s f} \cdot U_{d s} \cdot \Psi_{d f}+ \\
+k_{d s r} \cdot U_{d s} \cdot \Psi_{d r}+k_{q s} \cdot U_{q s} \cdot \Psi_{q s}+ \\
+k_{q s f} \cdot U_{q s} \cdot \Psi_{q f}+k_{q s r} \cdot U_{q s} \cdot \Psi_{q r} \\
q_{e m}=k_{d s} \cdot U_{q s} \cdot \Psi_{d s}+k_{d s f} \cdot U_{q s} \cdot \Psi_{d f}+ \\
+k_{d s r} \cdot U_{q s} \Psi_{d r}-k_{q s} \cdot U_{d s} \cdot \Psi_{q s}- \\
k_{q s f} \cdot U_{d s} \cdot \Psi_{q f}-k_{q s r} \cdot U_{d s} \cdot \Psi_{q r}
\end{array}\right\}
$$

It should be noted that the mentioned equations are written in the per unit system, for basic expressions the same units are taken as in the equations, which, for example, are given in $[9 ; 10 ; 11]$.

Coefficients $k_{d s}, k_{d s f}, k_{d s r}, k_{q s}, k_{q s f}, k_{q s r}, k_{d f}, k_{d f r}, k_{q f}, k_{q f r}$, $k_{d r}$ and $k_{q r}$ connect the values of currents of stator $i_{d s}, i_{q s}$, field windings $i_{d f}, i_{q f}$ and rotor windings $i_{d r}, i_{q r}$ with appropriate flux linkages.

They are easily defined from the matrix equality (direct and inverse matrices with inductive parameters of machine).

$\left[\begin{array}{cccccc}k_{d s} & 0 & k_{d s f} & 0 & k_{d s r} & 0 \\ 0 & k_{q s} & 0 & k_{q s f} & 0 & k_{q s r} \\ k_{d s f} & 0 & k_{d f} & 0 & k_{d f r} & 0 \\ 0 & k_{q s f} & 0 & k_{q f} & 0 & k_{q f r} \\ k_{d s r} & 0 & k_{d f r} & 0 & k_{d r} & 0 \\ 0 & k_{q s r} & 0 & k_{q f r} & 0 & k_{q r}\end{array}\right]=$

$$
=\left[\begin{array}{cccccc}
L_{d s} & 0 & M_{a d} & 0 & M_{a d} & 0 \\
0 & L_{q s} & 0 & M_{a q} & 0 & M_{a q} \\
M_{a d} & 0 & L_{d f} & 0 & M_{a d} & 0 \\
0 & M_{a q} & 0 & L_{q f} & 0 & M_{a q} \\
M_{a d} & 0 & M_{a d} & 0 & L_{d r} & 0 \\
0 & M_{a q} & 0 & M_{a q} & 0 & L_{q r}
\end{array}\right]^{-1}
$$

where $L_{d s}, L_{q s}, L_{d f}, L_{q f}, L_{q r}, L_{d r}$ - full inductances along the $d$ and $q$ axes to the corresponding stator, excitation and rotor windings [relative units]; $M_{a d}, M_{a q}-$ mutual inductances along the $d$ and $q$ axes [relative units].

In expression (4) the right part is a matrix of machine parameters in relative units, but it is necessary to have in mind that they are equal to the inductances of the machine in relative units, i.e. currents and flux linkages are connected by the values of the corresponding inductances, which in relative units are equal to the values of the passport parameters of the machine.

Structure of mathematical model of synchronous machine with excitation along the longitudinal and transverse axes $d$ and $q$. In this case the matrix form 
remains unchanged, except for control matrix $\left[\begin{array}{c}\boldsymbol{U}_{s} \\ \boldsymbol{U}_{f} \\ \boldsymbol{U}_{r}\end{array}\right]$, which turns into matrix $\left[\begin{array}{c}\boldsymbol{U}_{s} \\ \boldsymbol{U}_{f} \\ 0\end{array}\right]$, since for such machines the rotor has damper windings that are short-circuited.

Mathematical model of "classical" synchronous machine (having one field winding, located along the axis d), here besides the equality to zero $\boldsymbol{U}_{r}=0$, the following column-matrices will change:

$$
p \Psi_{f}=\left[\begin{array}{c}
p \Psi_{d f} \\
0
\end{array}\right] ; \quad \Psi_{f}=\left[\begin{array}{c}
\Psi_{d f} \\
0
\end{array}\right] ; \quad U_{f}=\left[\begin{array}{c}
U_{d f} \\
0
\end{array}\right]
$$

In addition, also diagonal matrices will change, which will occur in the form:

$\boldsymbol{B}_{f 1}=\left[\begin{array}{cc}-r_{d f} \cdot k_{d s f} & 0 \\ 0 & 0\end{array}\right] \quad \boldsymbol{B}_{f 2}=\left[\begin{array}{cc}-r_{d f} \cdot k_{d f} & 0 \\ 0 & 0\end{array}\right] ; \quad \boldsymbol{B}_{f 3}=\left[\begin{array}{cc}-r_{d f} \cdot k_{d r f} & 0 \\ 0 & 0\end{array}\right]$

As a result of equality $k_{q s s}=0$ and $k_{q f r}=0, \boldsymbol{A}_{s 2}$ and $\boldsymbol{C}_{r 2}$, will also be transformed, which will take the form:

$$
\boldsymbol{A}_{s 2}=\left[\begin{array}{cc}
-r_{s} \cdot k_{d s f} & 0 \\
0 & 0
\end{array}\right] \text { and } \boldsymbol{C}_{r 2}=\left[\begin{array}{cc}
-r_{d s} \cdot k_{d r f} & 0 \\
0 & 0
\end{array}\right]
$$

If the synchronous machine is made with permanent magnets, i.e. permanent magnets act as the exciter, then additionally the derivative matrix of flux linkages in expression (1) turns into the following matrix: $\left[\begin{array}{c}p \boldsymbol{\Psi}_{s} \\ 0 \\ p \boldsymbol{\Psi}_{r}\end{array}\right]$. And the voltage $\boldsymbol{U}_{d f}$, on which the flux linkage $\boldsymbol{\Psi}_{d f}$, depends, should be interpreted as a value that determines the coercive force of permanent magnets, or more exactly the magnetic energy value of permanent magnets, referred to the unit volume of permanent magnets [12].

Expression for the moment of "classical" synchronous machine will take the form:

$$
\begin{aligned}
m_{e m} & =\left(k_{q s}-k_{d s}\right) \Psi_{d s} \cdot \Psi_{q s}+k_{q s r} \cdot \Psi_{d s} \cdot \Psi_{q r} \\
& -k_{d s f} \cdot \Psi_{q s} \cdot \Psi_{d f}-k_{d s r} \cdot \Psi_{q s} \cdot \Psi_{d r}
\end{aligned}
$$

resistance matrix will appear in the form:

$$
\begin{aligned}
& {\left[\begin{array}{lllll}
k_{d s} & 0 & k_{d s f} & k_{d s r} & 0 \\
0 & k_{q s} & 0 & 0 & k_{q s r} \\
k_{d s f} & 0 & k_{d f} & k_{d f r} & 0 \\
k_{d s r} & 0 & k_{d f r} & k_{d r} & 0 \\
0 & k_{q s r} & 0 & 0 & k_{q r}
\end{array}\right]=} \\
& =\left[\begin{array}{llllr}
L_{d s} & 0 & M_{a d} & M_{a d} & 0 \\
0 & L_{q s} & 0 & 0 & M_{a q} \\
M_{a d} & 0 & L_{d f} & M_{a d} & 0 \\
M_{a d} & 0 & M_{a d} & L_{d r} & 0 \\
0 & M_{a q} & 0 & 0 & L_{q r}
\end{array}\right]^{-1}
\end{aligned}
$$

The structure of asynchronous machine model in this case: in matrix (1) the $3^{\text {rd }}$ row disappears and in diagonal matrix the $2^{\text {nd }}$ column and equation (1) transform into form:

$$
\left[\begin{array}{l}
p \boldsymbol{\Psi}_{s} \\
p \boldsymbol{\Psi}_{r}
\end{array}\right]=\left[\begin{array}{ll}
\boldsymbol{A}_{s 1} & \boldsymbol{A}_{s 3} \\
\boldsymbol{C}_{r 1} & \boldsymbol{C}_{r 3}
\end{array}\right]\left[\begin{array}{l}
\boldsymbol{\Psi}_{s} \\
\boldsymbol{\Psi}_{r}
\end{array}\right]+\left[\begin{array}{l}
\boldsymbol{U}_{s} \\
\boldsymbol{U}_{r}
\end{array}\right]
$$

Since the asynchronous machine is symmetrical in magnetic and electrical relation, then the parameters $k_{d s}=k_{q s} ; k_{d s r}=k_{q s s} ; k_{d r}=k_{q r}$ and $r_{d r}=r_{q r}$. Taking this into account, the submatrices are transformed into expressions,

$$
\begin{aligned}
& \boldsymbol{A}_{s 1}=\left[\begin{array}{cc}
-r_{S} \cdot k_{d s} & -\omega_{r} \\
\omega_{r} & -r_{s} \cdot k_{d s}
\end{array}\right]=-r_{S} \cdot k_{d s} \cdot \boldsymbol{E}-\boldsymbol{J} \cdot \omega_{r} \\
& \boldsymbol{A}_{s 3}=\left[\begin{array}{cc}
-r_{S} \cdot k_{d s r} & 0 \\
0 & -r_{S} \cdot k_{d s r}
\end{array}\right]=-r_{S} \cdot k_{d s r} \cdot \boldsymbol{E} \\
& \boldsymbol{C}_{r 1}=\left[\begin{array}{cc}
-r_{d r} \cdot k_{d s r} & 0 \\
0 & -r_{d r} \cdot k_{d s r}
\end{array}\right]=-r_{d r} \cdot k_{d s r} \cdot \boldsymbol{E} \\
& \boldsymbol{C}_{r 2}=\left[\begin{array}{cc}
-r_{d r} \cdot k_{d r} & 0 \\
0 & -r_{d r} \cdot k_{d r}
\end{array}\right]=-r_{d r} \cdot k_{d r} \cdot \boldsymbol{E}
\end{aligned}
$$

Substituting (7) for (6), one can obtain in expanded vector form an expression for derivative flux linkages of stator and rotor loops $\boldsymbol{\Psi}_{s}$ and $\boldsymbol{\Psi}_{r}$ :

$$
\left.\begin{array}{l}
p \boldsymbol{\Psi}_{S}=\boldsymbol{A}_{s 1} \cdot \boldsymbol{\Psi}_{S}+\boldsymbol{A}_{s 3} \cdot \boldsymbol{\Psi}_{r}+\boldsymbol{U}_{s}= \\
-r_{s} \cdot k_{d s} \cdot \boldsymbol{\Psi}_{S}-\boldsymbol{J} \cdot \omega_{r} \cdot \boldsymbol{\Psi}_{S}-r_{S} \cdot k_{d s r} \cdot \boldsymbol{\Psi}_{r}+\boldsymbol{U}_{S} \\
p \boldsymbol{\Psi}_{r}=\boldsymbol{C}_{r 1} \cdot \boldsymbol{\Psi}_{S}+\boldsymbol{C}_{r 3} \cdot \boldsymbol{\Psi}_{r}+\boldsymbol{U}_{r}= \\
r_{r} \cdot k_{d s r} \cdot \boldsymbol{\Psi}_{s}-r_{r} \cdot k_{d r} \cdot \boldsymbol{\Psi}_{r}+\boldsymbol{U}_{r}
\end{array}\right\}
$$

Coefficients $k_{d s}, k_{d r}, k_{d s r}$ can be determined from the equation:

$$
\left[\begin{array}{cccc}
k_{d s} & 0 & k_{d s r} & 0 \\
0 & k_{d s} & 0 & k_{d s r} \\
k_{d s r} & 0 & k_{d r} & 0 \\
0 & k_{d s r} & 0 & k_{d r}
\end{array}\right]=\left[\begin{array}{cccc}
L_{s} & 0 & M_{m} & 0 \\
0 & L_{s} & 0 & M_{m} \\
M_{m} & 0 & L_{r} & 0 \\
0 & M_{m} & 0 & L_{r}
\end{array}\right]^{-1}
$$

It should be noted that expressions (8) by form coincide with the equations of asynchronous machine mentioned in [13].

Expression for electromagnetic moment of asynchronous machine:

$$
m_{e m}=k_{d s r}\left(\Psi_{d s} \cdot \Psi_{q r}-\Psi_{q s} \cdot \Psi_{d r}\right)
$$

As it was already mentioned, in the model structure of AC machines as control parameters can be used $\boldsymbol{U}_{s}, f_{s}$ vector and frequency of the rotor winding voltage. Since the structure of the mathematical model of $\mathrm{AC}$ machines is based on the writing in the axes $d, q$ rotating at the rotor speed $\omega_{r}$, then there are no problems of modeling of voltages of field and rotor windings, as they are directly supplied from regulating devices (e.g., from the output of automatic excitation regulator of synchronous machine, either the output of frequency converter feeding the rotor winding for asynchronous machine).

This problem exists for stator winding. It is necessary to represent a vector of voltages, which supply stator winding of AC machine, whose components $\boldsymbol{U}_{d s}$ and $\boldsymbol{U}_{q s}$ are, naturally, written in axes $d$, $q$, rotating with rotor speed $\omega_{r}$, in such a form so that to be able to join control system with the electric AC machine (e.g. frequency converter). That is, it is necessary that they reflect the 
change (regulation) of the amplitude and frequency of the voltage supplying the stator winding. For this purpose the following transformations must be performed [12].

Location of coordinate axes of $\mathrm{AC}$ machines is reflected on the diagram (Fig.1). Here $\alpha_{0}, \beta_{0}$ - coordinate axes fixed in the space; $\alpha_{s}, \beta_{s}$ - coordinate axes rotating synchronously, with electric network frequency $\omega_{s} ; d, q-$ coordinate axes rotating at the rotor speed $\omega_{s}$. Angle between axes $\alpha_{s}, \beta_{s}$ and $\alpha_{0}, \beta_{0}$ we designate as $\alpha_{s}$, which is equal to $\alpha_{s}=\omega_{s} \cdot \tau$, where $\tau=\omega_{b a z} \cdot t=314 \cdot t-$ time in radians, $t$ - time in sec.

Angle between axes $d, q$ and fixed axes $\alpha_{0}, \beta_{0}$ we designate as $\alpha$, which is equal to $\alpha=\omega_{r} \cdot \tau$ and finally, angle $\theta=\alpha+\alpha_{s}-$ angle between axes $d, q$ and axes $\alpha_{s}, \beta_{s}$, which is called power angle.

If the stator voltage vector to place in the initial state at an angle $\pi / 4$ radians to the axes $\alpha_{s}, \beta_{s}$, then its projections on these axes will naturally be the same and equal to $U_{s \alpha 0}=U_{s \beta 0}=0.707 \cdot U_{s}$ (in relative units.).

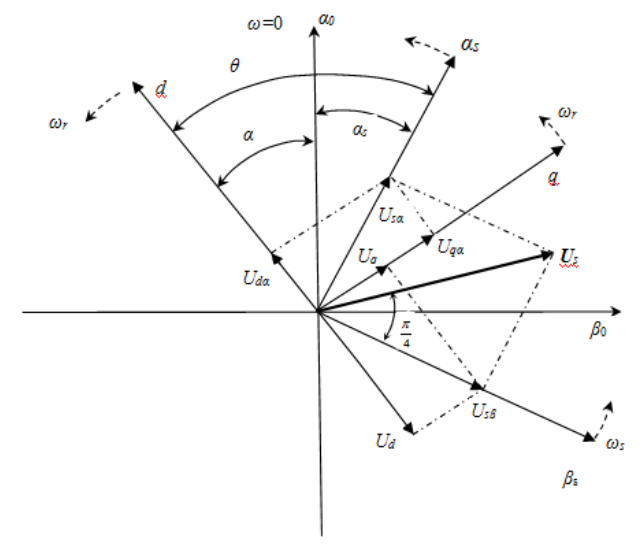

Fig.1. The location diagram of the coordinate axes in the model of electric machines of alternating current.

Projections of vectors $U_{s \alpha}$ and $U_{s \beta}$ on the axes $d, q$ in accordance with Fig. 1 can be written in the form:

$$
\left.\begin{array}{l}
U_{d \alpha}=U_{s \alpha} \cdot \cos \theta=U_{s \alpha} \cdot \cos \left(\alpha+\omega_{s} \cdot \tau\right) \\
U_{q \alpha}=U_{s \alpha} \cdot \sin \theta=U_{s \alpha} \cdot \sin \left(\alpha+\omega_{s} \cdot \tau\right) \\
U_{d \beta}=U_{s \beta} \cdot \sin \theta=U_{s \beta} \cdot \sin \left(\alpha+\omega_{s} \cdot \tau\right) \\
U_{q \beta}=U_{s \beta} \cdot \cos \theta=U_{s \beta} \cdot \cos \left(\alpha+\omega_{s} \cdot \tau\right)
\end{array}\right\}
$$

General projections of these components on the axes $d, q$ according to Fig. 1 will be in the form:

$$
\left.\begin{array}{l}
U_{d s}=U_{d \alpha}-U_{d \beta} \\
U_{q s}=U_{q \alpha}+U_{q \beta}
\end{array}\right\}
$$

Substituting expressions from (11) for (12) and taking into account that the vector $U_{s}$ just like the axes $\alpha_{s}, \beta_{s}$

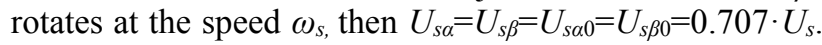
In addition, designating $k_{u s}=\frac{U_{s}}{U_{s 0}}$ and $k_{f s}=\frac{\omega_{s}}{\omega_{s 0}}=\frac{f_{s}}{f_{s 0}}$ where $U_{s 0}=1$ and $\omega_{s 0}=f_{s 0}=1$ [r.u.], we'll obtain:

$$
\begin{aligned}
& U_{d s}=0.707 \cdot k_{u s} \mid \cos \left(\alpha+k_{f s} \cdot \tau\right)-\sin \left(\alpha+k_{f s} \cdot \tau\right), \\
& \left.U_{q S}=0.707 \cdot k_{u s}\left[\sin \left(\alpha+k_{f S} \cdot \tau\right)+\cos \left(\alpha+k_{f S} \cdot \tau\right)\right]\right\}
\end{aligned}
$$

By means of simple transformations, expression (13) can be represented in a more convenient form:

$$
\left.\begin{array}{l}
U_{d s}=0.707 \cdot k_{u s}\left[\cos \left(k_{f_{S}} \cdot \tau\right) \cdot(\cos \alpha-\sin \alpha)-\right. \\
\left.-\sin \left(k_{f_{S}} \cdot \tau\right) \cdot(\cos \alpha+\sin \alpha)\right] \\
U_{q S}=0.707 \cdot k_{u s}\left[\cos \left(k_{f_{S}} \cdot \tau\right) \cdot(\cos \alpha+\sin \alpha)+\right. \\
+\sin \left(k_{f_{S}} \cdot \tau\right) \cdot(\cos \alpha-\sin \alpha]
\end{array}\right\}
$$

The control of the AC electric machine from the stator side is represented in (14). And for synchronous and asynchronous machines, this expression allows for taking into account the change and control of both the amplitude $k_{u s}$ of the voltage supplied to the machine and its frequency $k_{f s}$. Moreover, all other equations of $\mathrm{AC}$ machines remain written in the axes rotating at the rotor speed.

It should also be noted that in the absence of frequency converter in the stator circuit of $\mathrm{AC}$ electric machine, i.e. when the latter is connected directly to the electrical network $k_{u s}=k_{u s}=1$.

As it was said, $\boldsymbol{U}_{f}$ and $\boldsymbol{U}_{r}$ vectors can also act as control coordinates in the generalized cellular-matrix equations (1). As for the excitation voltage vector $\boldsymbol{U}_{f}$, in synchronous machine its components $\boldsymbol{U}_{d f}$ and $\boldsymbol{U}_{q f}$ along the axes $d, q$ assume the presence of longitudinal and transverse field windings and when presenting the equations written in the axes $d, q$, rotating at the rotor speed, in the structure of the equations they are constant and, if necessary, controlled values.

Regarding the voltage vector $\boldsymbol{U}_{r}$. For synchronous and asynchronous machines with short-circuited rotor it is equal to zero $\boldsymbol{U}_{r}=0$. For double-way feed asynchronous machines: at supply both from the stator side and the rotor side its value, naturally, is not equal to zero and in general case its components are written in the form [12]:

$$
\left.\begin{array}{l}
U_{d r}=k_{u r} \cdot \sin \left(k_{f r} \cdot \tau\right) \\
U_{q r}=k_{u r} \cdot \cos \left(k_{f r} \cdot \tau\right)
\end{array}\right\}
$$

where $U_{d r}, U_{q r}$ - components of rotor winding voltage, which are the output of frequency converter installed in rotor circuit, $k_{u r}=\frac{U_{r}}{U_{r_{0}}}-$ coefficient, taking into account the regulation of voltage amplitude, supplying the rotor winding, $k_{f r}=\frac{f_{r}}{f_{r_{0}}}-$ frequency of current at the output of the mentioned frequency converter, $U_{r 0}$ - rotor voltage amplitude in source mode (in relative units $U_{r 0}=1$ ).

Thus, expressions (13) and (15) allow for reproducing frequency control both from the stator side and the rotor side of electromechanical AC converters, i.e. together with equation (1) create a single universal structure of the mathematical model of controlled AC electric machines.

\section{Resuls}

The control of a controlled synchronous machine with one field winding along the $d$ axis has been investigated [12]. Just so a frequency-controlled synchronous machine with 2 windings along the axes $d, q$ respectively, can be studied [14]. 
Here study of controlled asynchronous machine has been conducted. In this case, the structure of the equations in vector form is represented by the expression (6), and the components of the stator voltage are modeled by the expression (14), and the rotary expression (15).

Parameters of machine: $x_{d}=4.878 ; r_{s}=0.01 ; x_{m}=4.8$; $r_{r}=0.31 ; k_{d s}=5.69 ; k_{d r}=5.66 ; k_{d s r}=5.56 ; 1 / T_{j}=0.005 ; x_{r}=4.9$.

Algorithm of solution of equations of asynchronous machine in general form is as follows:

$$
\mathrm{D}(\tau, \mathrm{Y})=\left[\begin{array}{l}
0.707 \cdot k_{u s} \cdot \cos \left(k_{f s} \cdot \tau\right) \cdot\left(\cos Y_{6}-\sin Y_{6}\right)-0.707 \cdot k_{u s} \\
\cdot \sin \left(k_{f s} \cdot \tau\right)\left(\cos Y_{6}+\sin Y_{6}\right)-Y_{5} \cdot Y_{2}-0.01 \cdot \\
\left(5.59 \cdot Y_{1}-5.56 \cdot Y_{3}\right) \\
0.707 \cdot k_{u s} \cdot \cos \left(k_{f s} \cdot \tau\right) \cdot\left(\cos Y_{6}+\sin Y_{6}\right)+0.707 \cdot k_{u s} \\
\cdot \sin \left(k_{f s} \cdot \tau\right) \cdot\left(\cos Y_{6}-\sin Y_{6}\right)+Y_{5} \cdot Y_{1}-0.01 \cdot \\
\left(5.69 \cdot Y_{2}-5.56 \cdot Y_{4}\right) \\
-k_{u r} \cdot \sin \left(k_{f r} \cdot \tau\right)-0.031 \cdot\left(5.66 \cdot Y_{3}-5.56 \cdot Y_{1}\right) \\
k_{u r} \cdot \cos \left(k_{f r} \cdot \tau\right)-0.031 \cdot\left(5.66 \cdot Y_{4}-5.56 \cdot Y_{2}\right) \\
0.005 \cdot\left(m_{t}\right)-0.005 \cdot \\
\cdot\left[Y_{1} \cdot\left(5.69 \cdot Y_{2}-5.56 \cdot Y_{4}\right)-Y_{2} \cdot\left(5.59 \cdot Y_{1}-5.56 \cdot Y_{3}\right)\right] \\
Y_{5}
\end{array}\right]
$$

On Fig.2 $(a, b, c, d)$ at $k_{u s}=k_{f s}=1$ (i.e. nominal values of amplitude and frequency of stator voltage) at the first stage at short-circuited rotor $\boldsymbol{U}_{r}=0$ in the range from 0 to $10^{3}$ radians the starting of generator with the value $m_{t}=$ 0.3 , is implemented and is set in the value $\omega_{r}=1.01$ (Fig.2,a). At $10^{3}$ radians the voltage is supplied to the rotor winding with steady-state value $k_{u s}=k_{f s}=-0.15$ (asynchronous machine with short-circuited rotor is transformed into double-way feed asynchronous machine). From $10^{3}$ to 2000 radians the rotating frequency becomes equal to $\omega_{r}=1.15$ (Fig.2,a). Electromagnetic moment is determined by the value $m_{t}$ and remains constant $m_{e m}=-0.3$ (Fig. $2, b$ ) ("minus" sign corresponds to generator mode). Curve of general active power, equal to sum of powers of stator and rotor circuits, is shown in the (Fig.2,c): its steady-state value varies from value $p_{w}=-0.3$ at short-circuited loop in the range from 0 to 1000 radians to value $p_{w}=0.34$ at $\tau>1000$ radians, when the rotor speed is increased to $\omega_{r}=1.15$ by means of regulation of frequency of current in the rotor winding of double-way feed machine. Reactive power (Fig.2, $d$ ) varies from value $q_{w}=0.24$ (consumes from network) to value $q_{w}=-0.218$ (delivers to network). After returning to source mode from 2000 radians to 3000 radians (i.e. when $k_{u s}=0$ ), is supplied. At control $k_{u s}=k_{f s}=0.15$ at 3000 radians the control equal to $\omega_{r}=0.85$ (Fig.2,a), the active power decreases from $p_{w}=-0.3$ to $p_{w}=-0.25$, respectively, and the reactive power, remaining in consumption mode, increases from $q_{w}=0.24$ to $q_{w}=0.49$, the moment $m_{e m}$ is unchanged and equal to $m_{e m}=0.3$.

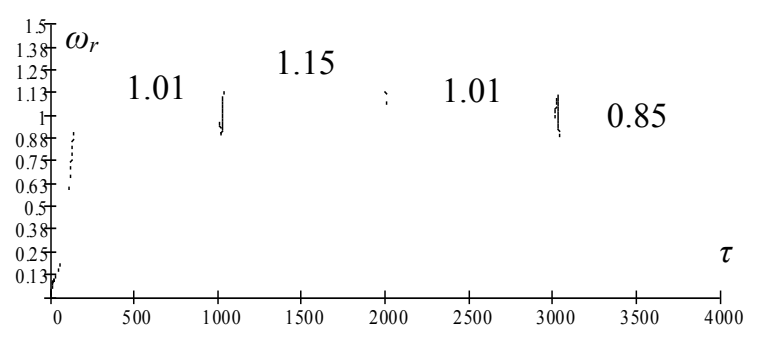

a)

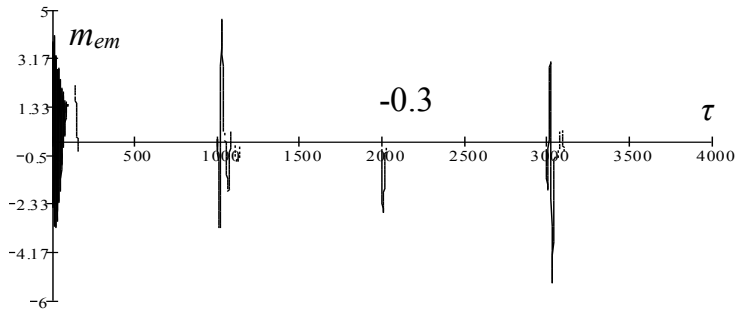

b)

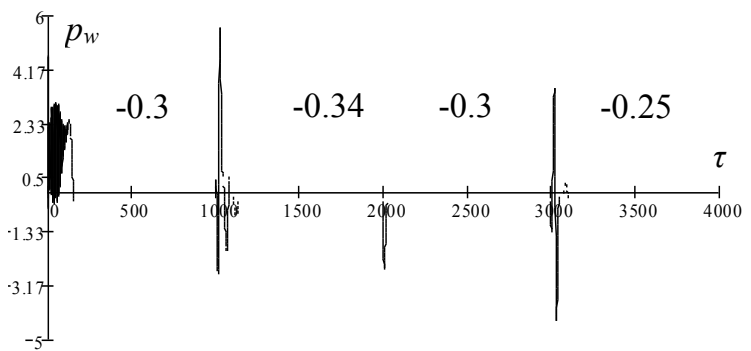

c)

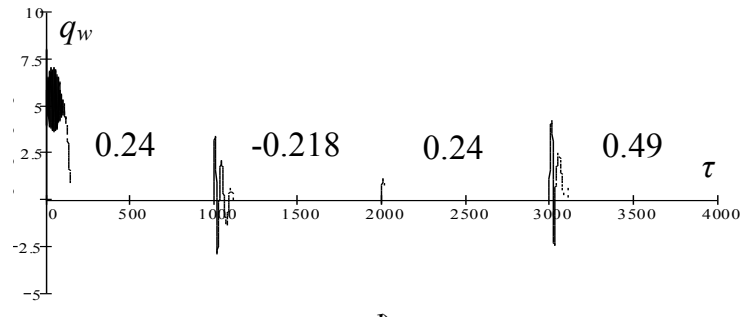

d)

Fig. 2. Fluctograms of change of operating parameters of an double-way feed asynchronous machine when using frequency regulation only from the rotor side with $k_{u r}=k_{f r}=-0,15$ and $k_{u r}=k_{f r}=+0,15$ with $k_{u s}=k_{f s}=1$.

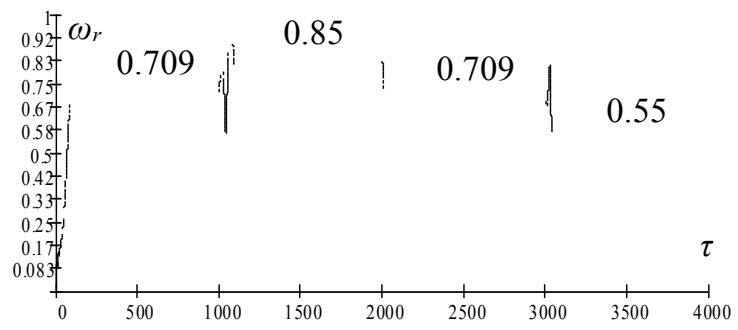

a) 


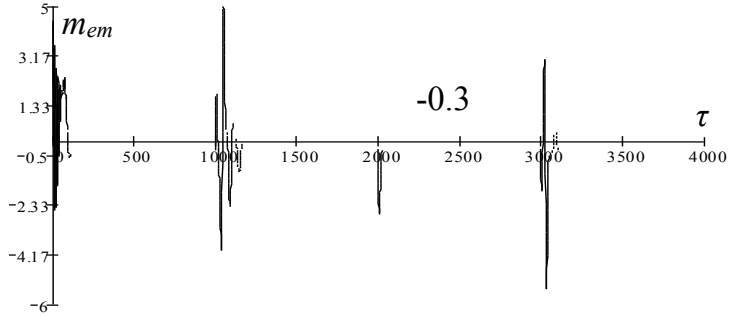

b)

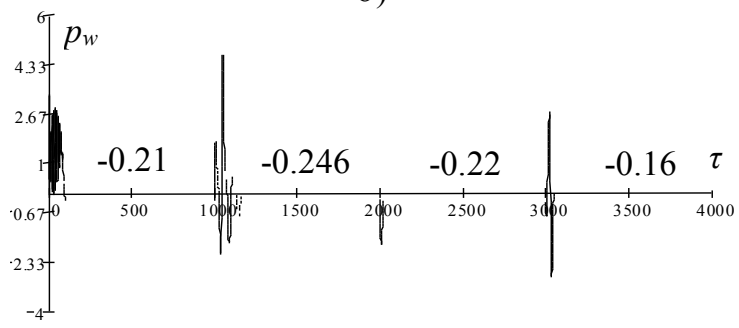

c)

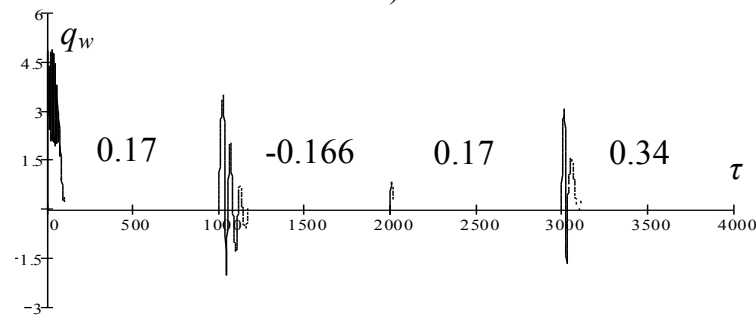

d)

Fig.3. Fluctograms of change of operating parameters of an double-way feed asynchronous machine when regulating voltage and frequency both from the stator side $k_{u s}=k_{f_{s}}=0.7$ and from the rotor side with $k_{u r}=k_{f r}=-0.15$ and $k_{u r}=k_{f r}=+0.15$.

Fluctograms of change of regime parameters of double-way feed asynchronous machine at control of voltage and frequency both from the stator side and from the rotor side are represented in Fig. $3(a, b, c, d)$. Here $k_{u s}=k_{f s}=0.7, k_{u s}=k_{f s}=-0.15$ at the first stage and $m_{t}=-0.3$. After starting the steady-state value is $\omega_{r}=0.7$ then at $k_{u s}=k_{f s}=-0.15$ (after 1000 radians) the $\omega_{r}$ becomes equal to $\omega_{r}=0.85$ (Fig. $3, a$ ), the $m_{e m}$ remains unchanged and equal to $m_{e m}=0.3$ (Fig. $3, b$ ). The active and reactive powers vary from $p_{w}=-0.21$ to $p_{w}=-0.246$, respectively, and $q_{w}=0.17$ (consumes from network) to $q_{w}=-0.166$ (delivers to network) (Fig. 3, $c$ and $d$ ).

There after source mode at 3000 radians at unchanged $k_{u s}=k_{f s}=0.7$ the value $k_{u s}=k_{f s}$, is regulated, which was set equal to $k_{u s}=k_{f s}=-0.15$. At that the rotor speed $\omega_{r}$ decreases to $\omega_{r}=0.55$. The active power accepts value $p_{e m}=-0.16$, and the reactive power in the consumption mode increases almost 2 times (as compared with the source mode) and becomes equal to $q=0.34$.

The given example of calculation confirms the efficiency of the universal mathematical model structure of controlled electric AC machines. It is known that main power control means for electromechanical $\mathrm{AC}$ transformers are frequency converters, which at present are implemented on completely controlled IGBTtransistors or GTO-thyristors with PWM control. The presented structure allows for taking into account in the mathematical model both amplitude and frequency of voltages supplying the stator and rotor circuits of $\mathrm{AC}$ machine.

If it is necessary to take into account a saturation, then this can be very simply done by existing methods: to operate by either saturated parameter values, or famous methods of their functional dependence [12]. As regards the consideration of harmonic composition of voltage at the outputs of frequency converters, their consideration also doesn't cause insurmountable difficulties - they can be taken into account in output voltage by means of harmonics factorized to Fourier series.

\section{References}

1. Kopylov I.P. Mathematical modeling of electric machines. Head School, Moscow, (1987)

2. Bespalov V.Y., Kotelenech N.F. Electric machines. Publ. Academy, 320 p., (2013)

3. Kazovskiy E.Y. Transients in AC electric machines. Publ. Academy of Sciences SSSR, Moscow, (1962)

4. Botvinnik M.M, Shakaryan Y.G. AC controlling machine. Sciences, Moscow, (1969)

5. Parviainen A., Niemela M., Pyrhonen J. Modeling of axial flux permanent-magnet machines // IEEE Transactions on Industry Applications, v.40, No. 5, pp. 1333-1340. (2004)

6. Simion A., Livadaru L., Munteanu A. Mathematical Model of the Three-Phase Induction Machine for the Study of Steady-State and Transient Duty Under Balanced and Unbalanced States. (2012)

7. Chiasson J. Modeling and High-Performance Control of Electrical Machines, IEEE Press, Wiley Interscience, Hoboken, USA, (2005)

8. Sul K.S. Control of Electric Machine Drive Systems. IEEE Press, Wiley Interscience, Hoboken, USA. (2011)

9. Sokolov N.I. Use of analogue computers in energy systems. Publ. Energy, Moscow, Leningrad. (1964)

10. Ong M.C. Dynamic Simulation of Electric Machinery using Matlab/Simulink,Prentice Hall, Jersey, USA. (1998)

11. Boldea I., Tutelea L. Electric Machines. Steady State, Transients and Design with MATLAB, CRC Press, Boca Raton, USA, (2010)

12. Mustafayev R.I., Hasanova L.H.. Modeling and research of operating modes of synchronous wind turbine generators with frequency control. Electricity, No. 7, pp. 34-40. (2010)

13. Kovach K.P., Rats I. Transients in AC machines. Trans. from German. Publ. Gosenergoizdat, Moscow, Leningrad. (1963)

14. Dyakonov V.P. Encyclopedia Mathcad 2001i and Mathcad 11.M.: SOLON-Press, 831 p., (2004) 